\title{
Quench Characteristics of the ATLAS Central Solenoid
}

\author{
R. J. M. Y. Ruber, Y. Makida, M. Kawai, S. Mizumaki, G. Olesen, H. H. J. ten Kate, and A. Yamamoto
}

\begin{abstract}
A thin superconducting solenoid has been constructed for ATLAS, one of the four LHC experiments at CERN. The single layer coil wound with an Al stabilized NbTi superconductor, with overall dimensions of $5.3 \mathrm{~m}$ length and $2.6 \mathrm{~m}$ diameter and operating at $7.6 \mathrm{kA}$ provides the $2 \mathrm{~T}$ magnetic field for the inner detector. The coil was successfully tested at the company before shipment and re-tested at CERN on surface in its final configuration before commencing the installation in the ATLAS cavern $100 \mathrm{~m}$ underground. The tests include an extensive study of the quench evolution and in particular the normal zone propagation through the coil windings and in the superconducting bus-lines. A special feature of this coil is the use of aluminum quench propagation strips glued to the windings inner surface. This design enhances the turn-to-turn normal zone propagation velocity, reaching up to $0.8 \mathrm{~m} / \mathrm{s}$, and limits the hot spot temperature to $110 \mathrm{~K}$. Along the conductor, normal zone propagation reaches velocities up to $14 \mathrm{~m} / \mathrm{s}$ at nominal current.
\end{abstract}

Index Terms-Detector magnet, hot spot temperature, $\mathrm{NbTi/Al}$ superconductors, normal zone propagation, quench.

\section{INTRODUCTION}

$\mathbf{T}$ HE CENTRAL Solenoid provides an axial magnetic field of $2 \mathrm{~T}$ in a $2.3 \mathrm{~m}$ diameter warm-bore for the ATLAS inner detector trackers [1]. The main parameters of the Central Solenoid are listed in Table I. The solenoid has a stored energy of $39 \mathrm{MJ}$, a cold mass of 5.6 tonnes and a thickness of $45 \mathrm{~mm}$ equivalent to 0.66 radiation length. For the coil to be this thin, it is wound with a single layer of specially developed high-strength aluminum stabilized NbTi superconductor [2]. A cross-section of the coil design is shown in Fig. 1. The coil is installed in the same cryostat as the barrel electro-magnetic calorimeter, the so-called barrel cryostat. It is attached to the inner warm vessel of the barrel cryostat by 23 triangular supports made of GFRP. These are arranged in such a way that one side of the coil is fixed and the other sliding in axial direction [3]. The cryostat is surrounded by the hadron calorimeter which also serves as a return yoke for the magnetic flux.

\section{AIR-CORE TEST SET-UP}

The Central Solenoid has been tested twice: at the factory site in Japan [4] and at the integration hall in CERN [5]. These tests were always performed in an air-core environment, whereas the final installation in the ATLAS detector will include an iron

Manuscript received September 20, 2005.

R. J. M. Y. Ruber, G. Olesen, and H. H. J. ten Kate are with CERN, CH-1211 Geneva 23, Switzerland (e-mail: ruber@cern.ch).

Y. Makida, M. Kawai, and A. Yamamoto are with KEK, Tsukuba 305-0801, Japan.

S. Mizumaki is with Toshiba Co., Tsurumi, Yokohama 230-0045, Japan.

Digital Object Identifier 10.1109/TASC.2006.873349
TABLE I

Main Parameters of THE Central SOLENOID

\begin{tabular}{ll}
\hline \hline Central Field & $2.0 \mathrm{~T}$ \\
Operating Current & $7600 \mathrm{~A}$ \\
Coil Size & $5.3 \mathrm{~m}(\mathrm{l}) \times 2.4 \mathrm{~m}(\Phi) \times 45 \mathrm{~mm}(\mathrm{t})$ \\
Warm Bore Diameter & $2.3 \mathrm{~m}$ \\
Wall Thickness & $0.66 \mathrm{X}_{0}$ \\
Stored Energy & $39 \mathrm{MJ}$ \\
Cold Mass & 5.6 tonnes \\
Inductance & $1.35 \mathrm{H}(1.27 \mathrm{H}$ when air core $)$ \\
Conductor Size & $30 \times 4.25 \mathrm{~mm}^{2}$ \\
Number of Strands & 12 \\
Strand Diameter & $1.25 \mathrm{~mm}$ \\
Conductor Area Ratio & $1: 0.9: 14(\mathrm{NbTi}$ Cu:Al $)$ \\
$\mathrm{I}_{c}$ at 5 T, 4.2 K & $22 \mathrm{kA}$ \\
Conductor RRR & $520-570$ \\
Cooling Mode & forced flow or thermo-syphon \\
\hline
\end{tabular}

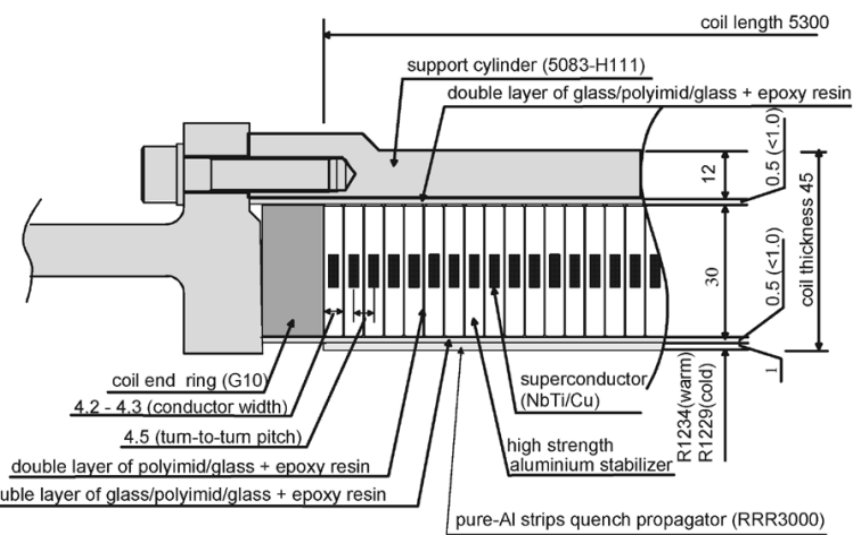

Fig. 1. Cross-section of the coil, showing support cylinder, end flange, conductor turns, insulation and pure aluminum strip quench propagators.

calorimeter that functions as a magnetic flux return yoke. For the test at the factory site, the solenoid was installed in a temporary cryostat, while the test at CERN was done with the final cryostat including the surrounding liquid argon calorimeter that functioned as part of the thermal screen. For both tests, the chimney (transfer line) connecting coil to proximity cryogenics and current leads was mounted horizontally, as opposed to its final vertical mounting position.

The main purpose of these tests was to verify operation of the solenoid at nominal field and study of the intrinsic safety of the design. In the ATLAS detector set-up the nominal operational field of the solenoid is $2.0 \mathrm{~T}$ at a current of $7.6 \mathrm{kA}$. In the air-core test set-up, a current of $8.0 \mathrm{kA}$ is required to reach the $2.0 \mathrm{~T}$ field. The maximum applied test current is $8.4 \mathrm{kA}$.

\section{INSTRUMENTATION AND QUENCH PROTECTION}

The solenoid is equipped with voltage taps, temperature sensors, superconducting quench detectors and pick-up coils [4]. Quench protection heaters (QPHT, $\phi=38 \mathrm{~mm}$ ) are installed 


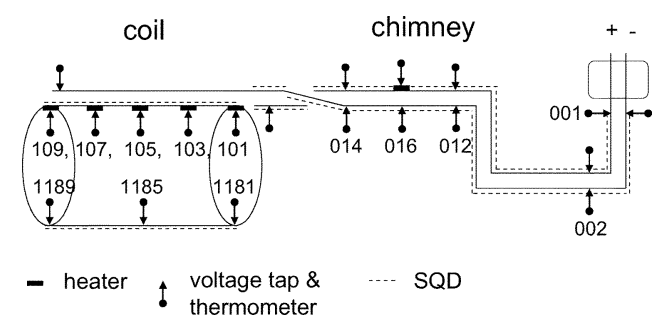

Fig. 2. Instrumentation layout of the coil and chimney including the numbers used to identify different points along bus-lines and windings.

near the conductor and may also be used to manually induce a quench. 72 High purity aluminum quench propagation strips, thickness $1 \mathrm{~mm}$ and width $100 \mathrm{~mm}$, are glued to the inner surface of the coil windings to enhance the turn-to-turn normal zone propagation velocity, as shown in Fig. 1, at a spacing of $4 \mathrm{~mm}$ or $38 \mathrm{~mm}$. Fig. 2 shows the location of instrumentation and heaters along the conductor in chimney and coil winding. Points 002, 012, 014 and 016 indicate voltage tap pairs on both positive and negative bus-lines with a typical length of some $300 \mathrm{~mm}$. As both bus-lines are clamped together, each point contains just a single temperature sensor. The points marked 101 through 109 at the top of the winding $\left(0^{\circ}\right)$ contain heaters, temperature sensors and voltage tap pairs. The heaters are normally connected to the safety system, but were in some cases disconnected and used to induce a quench. Points 1181,1185 and 1189 are at the bottom of the windings $\left(180^{\circ}\right)$ and contain temperature sensors and voltage tap pairs. The voltage tap pairs (at top and bottom) cover a conductor length of $90^{\circ}$ along the circumference of a single turn.

A quench detection unit was used to trigger the data acquisition units and to fire the heaters described above in case they were connected to a quench protection unit. The quench detection unit applies a $1.2 \mathrm{~s}$ window in which an input signal must be above threshold setting before releasing the quench detection trigger. The pick-up coils at each end of the coil winding are used, in combination with a voltage tap pair for the total coil voltage, as a trigger for quench detection. In a similar fashion several superconducting quench detectors (SQD) are installed along bus-lines and top and bottom of the coil winding. These are tiny superconducting wires quenching simultaneous with the conductor and thus also useful as quench detection trigger and monitor of the quench propagation [6].

\section{NORMAL ZONE PROPAGATION}

\section{A. Training Quenches}

There have been four training quenches. Two during the factory test, both slightly below the nominal operational current (7016 and 7546 A). And two during the on-surface test at CERN after transport from Japan and integration into the final cryostat, with both quenches at currents far above the nominal operational current (7964 and 8112 A). Fig. 3 summarizes the quenches. The first training quench was at $92 \%$ of the nominal current. There is no observed degradation after thermal cycling, transport and integration work.

From the voltage tap signal timing we can determine that the quench at the lowest current during the factory test and the

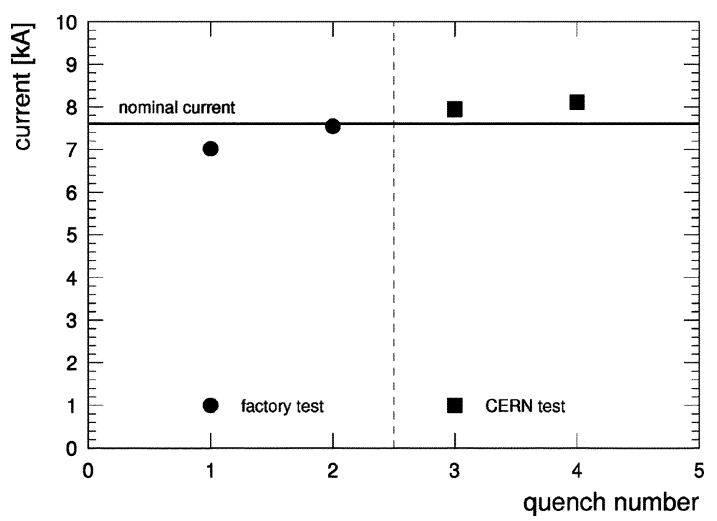

Fig. 3. Training quenches.

CERN test were both times at the end opposite to the chimney connection, likely in the last few turns facing the end flange (point 109). The second quench was in the last few turns at the opposite coil end (point 101) during both tests. During the tests at CERN, the pick-up coils were being monitored. The pick-up coil signal started to rise 0.2 to $0.3 \mathrm{~s}$ before the voltage tap signal, indicating the time required for the normal zone to propagate to the voltage taps. With a normal zone propagation velocity in the order of $15 \mathrm{~m} / \mathrm{s}$ this indicates that the quench origin was located within some $3 \mathrm{~m}$ or a quarter turn from the voltage taps in the last or last few turns facing the end flange. A quench in this area is likely due to the relatively large shear stress and eventually tensile stress in the outer turns.

\section{B. Bus-Lines}

A quench of the bus-lines in the chimney was induced by firing the heater at position 016. This heater is located on the positive bus-line. Data is available from a single test at $6 \mathrm{kA}$. The normal zone propagated with a speed of $2.88 \pm 0.06 \mathrm{~m} / \mathrm{s}$ to point 012 , and with a speed of $1.97 \pm 0.04 \mathrm{~m} / \mathrm{s}$ to point 014 which includes, besides a horizontal section, also a vertical section. An average propagation speed of $2.4 \pm 0.6 \mathrm{~m} / \mathrm{s}$ at $6 \mathrm{kA}$ is observed.

A delay of $0.54 \pm 0.01 \mathrm{~s}$ appeared between a rising voltage tap signal on the positive and negative bus-line at point 016 following the firing of the heater. This delay may be fully attributed to the insulation layers between the two bus-lines: 2 times 4 layers of Upilex/Glass tape. When the quench front arrives at voltage taps 012 and 014 the timing difference between positive and negative bus-lines is reduced to $0.15 \pm 0.01 \mathrm{~s}$ and 0.13 $\pm 0.01 \mathrm{~s}$ respectively. As the distance from point 016 to 014 is $10 \%$ longer than to point 012 , it shows that the delay between the bus-lines due to the insulation layers only has a significant effect at the beginning of the quench.

The signal of the SQD on the positive bus-line starts rising a few tenths of a second before the voltage taps signal. In this case the SQD signal is influenced by the heater location which is, like the SQD, outside of the insulation of the bus-line, on top of the SQD. The signal of the SQD on the negative bus-line starts rising almost 2 seconds after the voltage taps signal of the negative bus-line. Here we have the effect of the bus-line insulation (4 layers of Upilex/Glass tape) plus ground insulation (2 layers of Glass/Upilex/Glass tape). 


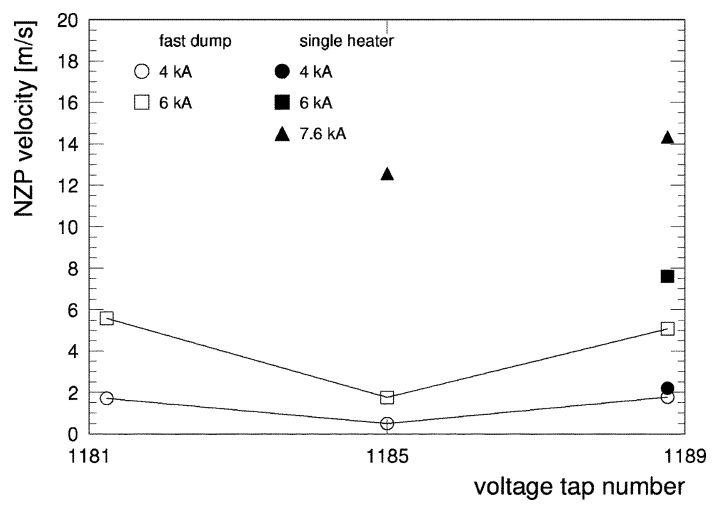

Fig. 4. Normal zone propagation velocity in the coil windings. The velocity is calculated between the heater at the top and the voltage tap at the bottom of the coil windings.

The advantage of using SQDs is their galvanic isolation from the conductor and a high signal-to-noise ratio. However, for this specific application the voltage signal over the bus-line gives clearly a faster response as quench detection trigger than the SQDs. The bus-line voltage reaches a threshold of $100 \mathrm{mV}$ within $0.7 \mathrm{~s}$ while the SQDs do not reach this threshold until nearly 4 to $5 \mathrm{~s}$ after quench start (for positive and negative bus-line SQD respectively).

\section{Coil Windings}

For the coil windings, data is available where the quench started at one single location or was initiated by all 5 protection heaters simultaneously. For the second case there are measurements at 4 and $6 \mathrm{kA}$. For the first case data is available between 4 and $7.6 \mathrm{kA}$.

Fig. 4 shows the normal zone propagation (NZP) velocity along the conductor. The data is collected quenches induced by a single heater or so called fast dumps in which all 5 heaters at the top of the coil winding are fired simultaneously. The NZP velocity was calculated between the heater at the top and the voltage tap at the bottom of the coil winding. The propagation velocity along the conductor at the coil ends is in the order of $2.2 \mathrm{~m} / \mathrm{s}$ for a single heater induced quench and $1.7 \pm 0.1 \mathrm{~m} / \mathrm{s}$ for a fast dump at $4 \mathrm{kA}$. At $6 \mathrm{kA}$ the velocity increases to $7.6 \pm 0.2$ and $5.3 \pm 0.3 \mathrm{~m} / \mathrm{s}$ respectively. The data for $7.6 \mathrm{kA}$ indicates a propagation speed of $14.3 \pm 0.7 \mathrm{~m} / \mathrm{s}$ at a coil end and $12.6 \pm$ $0.2 \mathrm{~m} / \mathrm{s}$ at the coil center.

During the tests where just a single heater is fired, the turn-toturn NZP velocity perpendicular to the NZP along the conductor may be measured. A graphical overview of the data is plotted in Fig. 5. At $7.6 \mathrm{kA}$ this speed is $0.31 \mathrm{~m} / \mathrm{s}$ for the first quarter of the coil windings and $0.82 \pm 0.02 \mathrm{~m} / \mathrm{s}$ for the second quarter.

\section{Aluminum Strip Quench Propagators}

The increase in the turn-to-turn NZP velocity is an effect of eddy current heating and heat distribution by the pure aluminum strips. The strips provide a very efficient distribution of the heat from the quenched area to the rest of the coil [7]. The pure aluminum strips are attached in axial direction along the coil windings. Their effect on the heat distribution and minimization of the hot spot temperature was proven previously [8]. The effect on the NZP velocity is shown by the thermal conductivity of the

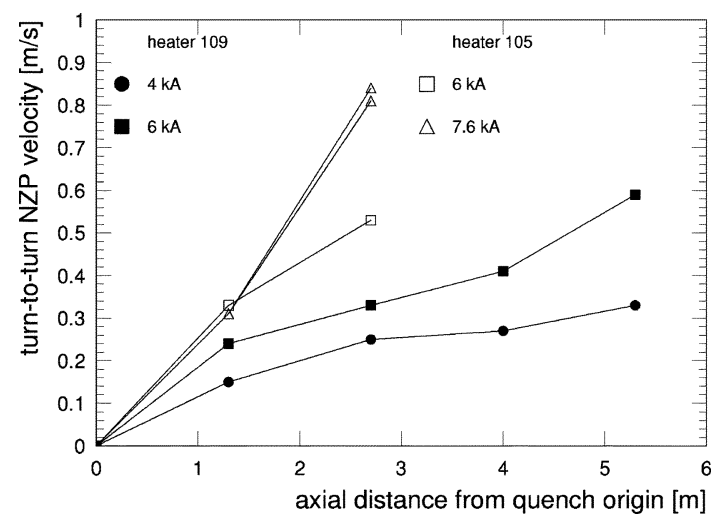

Fig. 5. Turn-to-turn normal zone propagation velocity as function of the axial distance along the coil windings from the quench origin. Data is shown for several currents and different quench origins by the heater located at either point 105 or 109.

strips. The heat flow $\dot{Q}$ through two materials in parallel (strips and windings), over a length $l$ with cross-section area $A$ due to a temperature difference $\Delta T$ is given by

$$
\dot{Q}=\left(k_{1} \cdot \frac{A_{1}}{l_{1}}+k_{2} \cdot \frac{A_{2}}{l_{2}}\right) \cdot \Delta T
$$

with $k$ the thermal conductivity. Both heat flows are also in series with the insulation material. The effective $k$ may be calculated as

$$
k=\frac{l_{a}+l_{b}}{\frac{l_{a}}{k_{a}}+\frac{l_{b}}{k_{b}}} .
$$

Hence, the influence of the pure aluminum strips to the heat flow increases with distance. For example, over a distance of $1.3 \mathrm{~m}$, the thermal conductivity via the strips becomes $106 \mathrm{~W} / \mathrm{mK}$ and increases to $208 \mathrm{~W} / \mathrm{mK}$ over a distance of $2.6 \mathrm{~m}$. This may be compared with a turn-to-turn thermal conductivity of the windings of $1.1 \mathrm{~W} / \mathrm{mK}$. Here we assume for the strips that $k=$ $5 \mathrm{~kW} / \mathrm{mK}$ at $5 \mathrm{~K}$ and $2 \mathrm{~T}$, and that the thermal conductivity of the insulation is dominated by layers of $20 \mu \mathrm{m}$ Upilex films with $k=1 \times 10^{-2} \mathrm{~W} / \mathrm{mK}$ at $4.5 \mathrm{~K}$ [9].

Thus the pure aluminum strips clearly have an important contribution to the heat distribution and turn-to-turn normal zone propagation velocity. This effect becomes very significant with increasing distance to the quench origin.

\section{TEMPERATURE DISTRIBUTION}

The temperature evolution in the coil windings is a function of the initial stored energy and its distribution during a quench. The highest temperatures are reached when the coil quenches at the end of the windings as then the quench can only propagate in one direction. Fig. 6 summarizes the hot spot temperature of the coil windings as a function of the current for different quench origins. It also distinguishes for data where the QPHTs are or are not activated.

To minimize the maximum, so called hot spot, temperature of the windings, active and passive techniques are used. The passive technique consists of the highly pure aluminum strip 


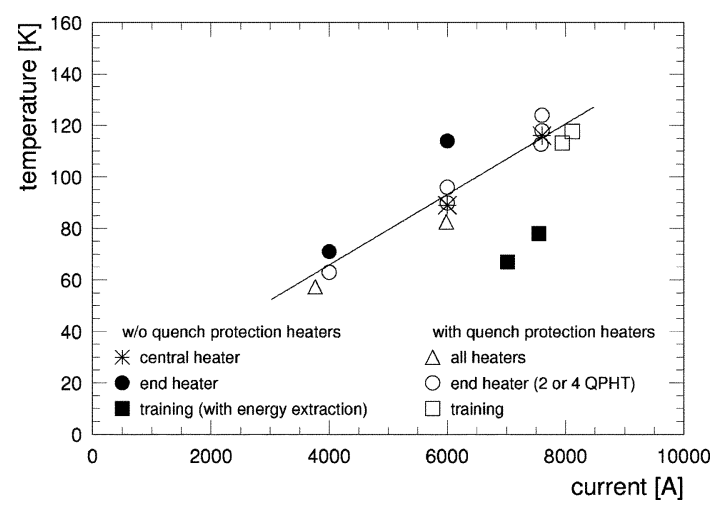

Fig. 6. Hot spot temperature as function of the coil current with and without the quench protection heaters (QPHT) activated and for different quench ignition points. Energy extraction indicates usage of a different external protection system [4].

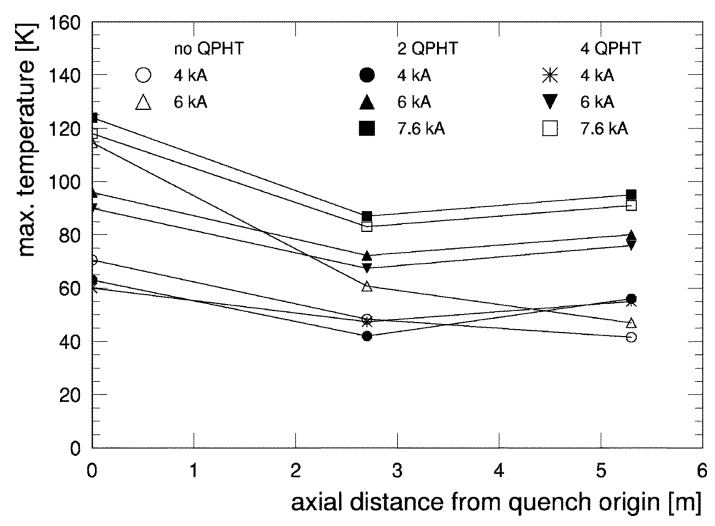

Fig. 7. Temperature evolution as function of the axial distance from the quench origin, for different currents and with no, 2 or 4 QPHTs. Quench origin is at the point 109 heater.

quench propagators (RRR-3000) described above, that are installed axially along the windings to increase the axial heat conduction capacity. The active technique consists of firing the heaters installed along the top of the coil windings. The effect of these QPHTs is shown in Fig. 7. All data plotted in this figure are for quenches starting at a coil end. For a quench at $6 \mathrm{kA}$, the hot spot temperature decreases sharply from 115 to $96 \mathrm{~K}$ when using 2 QPHTs and to $90 \mathrm{~K}$ with 4 QPHTs. Simultaneously the temperature difference over the coil windings drops from $68 \mathrm{~K}$ to $16 \mathrm{~K}$ with 2 QPHTs and to $14 \mathrm{~K}$ with 4 QPHTs. At $7.6 \mathrm{kA}$ and 2 heaters operational, the hot spot temperature becomes $124 \mathrm{~K}$ and the temperature gradient $29 \mathrm{~K}$. With 4 heaters operational the hot spot temperature is limited to $113 \mathrm{~K}$ and the temperature gradient to $27 \mathrm{~K}$.

\section{CONCLUSION}

A test current of up to $8.4 \mathrm{kA}$ was applied and the coil went through almost thirty quench cycles including four spontaneous quenches. The normal zone propagation velocity along the conductor varies between $2.4 \mathrm{~m} / \mathrm{s}$ in the bus-lines to $14 \mathrm{~m} / \mathrm{s}$ in the coil windings. The turn-to-turn normal zone propagation velocity reaches up to $0.8 \mathrm{~m} / \mathrm{s}$. With a stored energy of $39 \mathrm{MJ}$ and 5.6 tonnes cold mass the maximum temperature in the windings was limited to about $110 \mathrm{~K}$. The combination of superconducting quench detectors, voltage taps and pick-up coils provides a good and fast quench detection as required for firing the protection heaters.

The design of the ATLAS Central Solenoid provides a reliable and safe detector solenoid. The use of pure aluminum strips and an active quench protection heater system enhances the normal zone propagation velocity and heat distribution during quench. The heater system, triggered by a quench detection unit, significantly reduces the windings hot spot temperature during a quench.

\section{ACKNOWLEDGMENT}

The authors would like to thank their colleagues in the ATLAS collaboration, especially those from KEK, Toshiba, and CERN for all their efforts in preparing the installation and test setup.

\section{REFERENCES}

[1] A. Yamamoto et al., "Progress in ATLAS central solenoid magnet," IEEE Trans. Appl. Supercond., vol. 10, p. 353, 2000.

[2] K. Wada et al., "Development of high-strength and high-RRR aluminum-stabilized superconductor for the ATLAS thin solenoid," IEEE Trans. Appl. Supercond., vol. 10, p. 373, 2000.

[3] S. Mizumaki et al., "Fabrication and mechanical performance of the ATLAS central solenoid," IEEE Trans. Appl. Supercond., vol. 12, p. 416, 2002.

[4] Y. Makida et al., "Quench protection and safety of the ATLAS central solenoid," IEEE Trans. Appl. Supercond., vol. 12, p. 407, 2002.

[5] R. J. M. Y. Ruber et al., "ATLAS superconducting solenoid on-surface test," IEEE Trans. Appl. Supercond., vol. 15, p. 1283, 2005.

[6] A. Dudarev et al., "Quench propagation and detection in the superconducting bus-bars of the ATLAS magnets," IEEE Trans. Appl. Supercond., vol. 10 , p. 381, 2000.

[7] A. Yamamoto et al., "Conceptual design of a thin superconducting solenoid for particle astrophysics," IEEE Trans. Magn., vol. 24, p. 1421, 1988.

[8] Y. Makida et al., "Development of a thin superconducting solenoid for particle astrophysics," Adv. Cryogenic Eng., vol. 37A, p. 401, 1992.

[9] M. Barucci et al., "Low temperature thermal conductivity of Kapton and Upilex," Cryogenics, vol. 40, p. 145, 2000. 